\title{
Solving Coplanar Power-Limited Orbit Transfer Problem by Primer Vector Approximation Method
}

\author{
Weijun Huang \\ Department of Mechanical and Aerospace Engineering, University of Missouri, Columbia, MO 65211, USA \\ Correspondence should be addressed to Weijun Huang, whfy6@mail.missouri.edu
}

Received 1 November 2011; Revised 12 January 2012; Accepted 16 January 2012

Academic Editor: Alessandro A. Quarta

Copyright () 2012 Weijun Huang. This is an open access article distributed under the Creative Commons Attribution License, which permits unrestricted use, distribution, and reproduction in any medium, provided the original work is properly cited.

The coplanar orbit transfer problem has been an important topic in astrodynamics since the beginning of the space era. Though many approximate solutions for power-limited orbit transfer problem have been developed, most of them rely on simplifications of the dynamics of the problem. This paper proposes a new approximation method called primer vector approximation method to solve the classic power-limited orbit transfer problem. This method makes no simplification on the dynamics, but instead approximates the optimal primer-vector function. With this method, this paper derives two approximate solutions for the powerlimited orbit transfer problem. Numerical tests show the robustness and accuracy of the approximations.

\section{Introduction}

Most trajectory optimization problems are nonlinear problems with no analytic solutions. However, to the coplanar power-limited orbit transfer in the classical inverse-square gravity field, many researchers have proposed approximate solutions, for example, Edelbaum [1-4], Zee [5], Marinescu [6], Marec and Vinh [7], Haissig et al. [8], Kechichian's [9], and Casalino and Colasurdo's [10]. These proposed solutions are built on assumptions about the transfer scenarios. For example, the solution of [6] assumes the transfer is in a close range, the solutions of $[2,4-6]$ assume the transfer happens in a long duration, and the solutions of $[1,3,7-11]$ assume the admissible control to be within a rather limited set.

An approximate solution to an optimal control problem implies both the approximation of the control policy and the approximation of the dynamics. However, we automatically approximate the optimal control policy as well, when we approximate the dynamics. The mentioned references more or less approximate the dynamics. One potential problem with approximating dynamics is that, once the assumptions are violated the obtained control will be infeasible. From a software point of view, an infeasible control produces unexpected results and might cause the software to crash. Therefore, a more robust approximation method for optimal control problem should be to purely make approximations on the optimal control, not the dynamics.

A popular method for generating feasible transfer trajectories is the shape-based method [12-15]. In its essence, this method generates feasible control without any compromise on the dynamics. Though there are different variates of the shape-based method, as far as the author knows, there is no theoretical research to address the connection between the real optimal solution and the trajectory generated by the shape-based method.

This paper proposes an innovative approximation method-the primer vector approximation method, which combines the advantages of the approximation method and the shape-based method and uses feasible control to approximate the optimal control. The method reformulates the classic transfer problem with a nonlinear transformation from Carter and Humi [16]. The purpose of this reformulation is to put all the nonlinear terms to the coefficients of the control variables. Thus the optimal control vector, called primer vector, of the new formulation can be analyzed and approximated without affecting the dynamics. To demonstrate this method, this paper derives two approximate solutions for both low-thrust close-range transfer and low-thrust longduration transfer. 


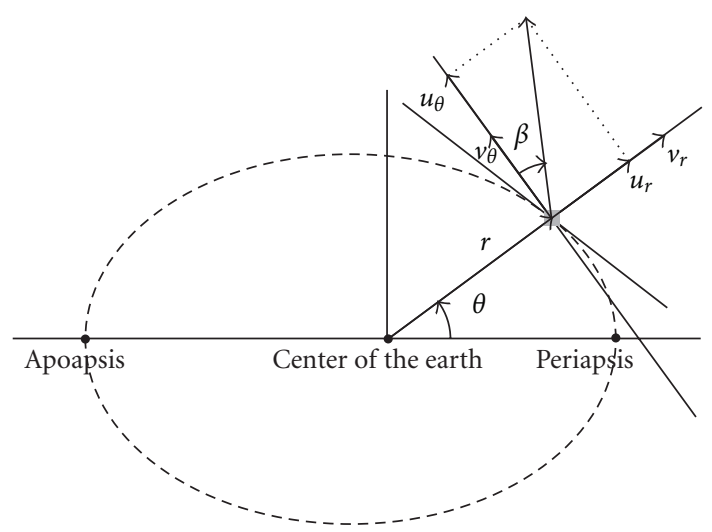

Figure 1: Definition of the polar coordinate.

Four transfer scenarios are designed to test the two approximate solutions numerically. The tests show that the approximations are close to the optimal solutions when the scenarios are within the assumptions of the approximations. More importantly, the solutions are feasible even when the scenarios are far away from the assumptions of the approximations. Theoretically, both approximations can generate feasible transfer control between any two kinds of orbits, including hyperbolic and parabolic orbit.

The remaining part of this paper is organized in the following way. The second section is about the formulations of the coplanar power-limited orbit transfer problem. The third section introduces the primer vector approximation method and derives two approximate solutions. The fourth section numerically tests the two solutions. A conclusion is made in the fifth section.

\section{Formulations of Coplanar Power-Limited Orbit Transfer Problem}

2.1. Polar Coordinate Formulation. The polar coordinate system used in this paper is presented in Figure 1. The meanings of the symbols are given in Nomenclature.

Mathematically, the dynamics of a controlled satellite in an inverse-square gravity field can be described as

$$
\begin{gathered}
\ddot{r}-r \dot{\theta}^{2}=-\frac{\mu}{r^{2}}+u_{r}, \\
\ddot{\theta} r+2 \dot{\theta} \dot{r}=u_{\theta} .
\end{gathered}
$$

Set $v_{r}=d r / d t$ and $v_{\theta}=\dot{\theta} r$. The two second-order ordinary differential equations (ODEs), (1), are equivalent to the following four first-order ODEs:

$$
\begin{gathered}
\dot{r}=v_{r}, \\
\dot{\theta}=\frac{v_{\theta}}{r}, \\
\dot{v}_{r}=\frac{v_{\theta}^{2}}{r}-\frac{\mu}{r^{2}}+u_{r}, \\
\dot{v}_{\theta}=-\frac{v_{\theta} v_{r}}{r}+u_{\theta} .
\end{gathered}
$$

Set $\mathbf{u}=\left(u_{r}, u_{\theta}\right)^{T}$. If a transfer start from time $t_{0}$ to time $t_{f}$, the objective function of the coplanar power-limited orbit transfer problem is

$$
J(\mathbf{u})=\frac{1}{2} \int_{t_{0}}^{t_{f}} \mathbf{u}^{T} \mathbf{u} d t
$$

The initial orbit at $t_{0}$ is defined by a vector $\mathbf{x}_{0}=$ $\left(r_{0}, \theta_{0}, v_{r 0}, v_{\theta 0}\right)^{T}$ and the final orbit at $t_{f}$ is defined by a vector $\mathbf{x}_{f}=\left(r_{f}, \theta_{f}, v_{r f}, v_{\theta f}\right)^{T}$. A free-time rendezvous problem is to find the optimal control function $\mathbf{u}^{*}$, which minimizes the objective function, (3), and transfers a spacecraft from the original position $\mathbf{x}_{0}$ at time $t_{0}$ to the final position $\mathbf{x}_{f}$ at the optimal arriving time $t_{f}^{*}$. This problem is the simplest problem to introduce the primer vector approximation method. A more common power-limited transfer problem unbounded thrust is the fixed-time rendezvous problem, the solution to which can be found by adding a time constraint on the approximate solution to the free-time rendezvous problem. We can adopt the method in Novak's [15] paper to use a solution to the free-time rendezvous problem as a basis for the solution to the related fixed-time rendezvous problem. This paper, however, focuses only on the free-time rendezvous problem.

To numerically solve the free-time rendezvous problem, we can use the indirect shooting method, which first transforms the problem into a two-point boundary value problem (TPBVP) and then solves the TPBVP with the shooting technique. To setup the TPBVP, we write down the augmented Hamiltonian of the optimal problem as follows:

$$
\begin{aligned}
H= & -\frac{1}{2} \mathbf{u}^{T} \mathbf{u}+\lambda_{r} v_{r}+\lambda_{\theta} \frac{v_{\theta}}{r}+\lambda_{v r}\left(\frac{v_{\theta}^{2}}{r}-\frac{\mu}{r^{2}}+u_{r}\right) \\
& +\lambda_{\theta}\left(-\frac{v_{\theta} v_{r}}{r}+u_{\theta}\right) .
\end{aligned}
$$

The adjoint ODEs of the problem are

$$
\begin{gathered}
\dot{\lambda}_{r}=\frac{\lambda_{\theta} v_{\theta}}{r^{2}}+\lambda_{v r}\left(\frac{v_{\theta}^{2}}{r^{2}}-2 \frac{\mu}{r^{3}}-\frac{\lambda_{v \theta} v_{r} v_{\theta}}{r^{2}}\right), \\
\dot{\lambda}_{\theta}=0, \\
\dot{\lambda}_{v r}=-\lambda_{r}+\frac{\lambda_{v \theta} v_{\theta}}{r}, \\
\dot{\lambda}_{v \theta}=\frac{1}{r}\left(\lambda_{v \theta} v_{r}-\lambda_{\theta}-2 \lambda_{v r} \lambda_{\theta}\right) .
\end{gathered}
$$

The optimal control is $\mathbf{u}^{*}=\mathbf{p}$, where $\mathbf{p}=\left(\lambda_{v r}, \lambda_{v \theta}\right)^{T}$ and is called primer vector. A common shooting method will shoot the optimal arrival time $t_{f}^{*}$ and the values of the four variables $\lambda_{r}\left(t_{0}\right), \lambda_{\theta}\left(t_{0}\right), \lambda_{v r}\left(t_{0}\right)$, and $\lambda_{v \theta}\left(t_{0}\right)$ until the shooting functions $\mathbf{x}_{(}\left(t_{f}^{*}\right)=\mathbf{x}_{f}$ and transversality condition $H\left(t_{f}^{*}\right)=0$ are satisfied. At each iteration of the shooting process, there are eight ODEs, (2) and (5), to solve. The shooting process will be time-consuming if the initial guess is poor or the number of the transfer revolutions is large.

In this paper, the indirect shooting method with the polar coordinate formulation will be used to generate optimal solutions for the four tests in the fourth session. 
2.2. A New Formulation. The polar formulation of the power-limited orbit transfer problem has nonlinear terms in the dynamic equations and adjoint equations. A new formulation of the power-limited orbit transfer problem will be derived here with a nonlinear transformation from Carter and Humi [16]. This transformation will transfer the state variables $\left(r, \theta, v_{r}, v_{\theta}\right)$ with respect to time $t$ to new state variables $\left(y_{1}, y_{2}, y_{3}\right)$ with respect to polar angle $\theta$.

Use $(\cdot)^{\prime}$ and $(\cdot)^{\prime \prime}$ to represent the first and second derivative operations with respective to the polar angle $\theta$. Set $h=$ $\dot{\theta} r^{2}$. Thus $\dot{\theta}=h / r^{2}$ and $t^{\prime}=d t / d \theta=r^{2} / h$. The new state variables are defined as $y_{1}=1 / r, y_{2}=-r^{\prime} / r^{2}$, and $y_{3}=\mu / h^{2}$. The mapping from the new variables $\left(y_{1}, y_{2}, y_{3}\right)$ back to the variables in the polar coordinate is

$$
\begin{gathered}
r=\frac{1}{y_{1}}, \\
v_{r}=-y_{2} \sqrt{\frac{\mu}{y_{3}}}, \\
v_{\theta}=y_{1} \sqrt{\frac{\mu}{y_{3}}}, \\
t=\theta_{0}+\int_{\theta_{0}}^{\theta} \frac{1}{y_{1}^{2}} \sqrt{\frac{y_{3}}{\mu}} d \theta .
\end{gathered}
$$

From the chain rule, we have

$$
\begin{gathered}
\ddot{\theta}=\left[\frac{h^{\prime}}{r^{2}}-2 \frac{h}{r^{3}} r^{\prime}\right] \frac{h}{r^{2}}, \\
\dot{r}=r^{\prime} \frac{h}{r^{2}}, \\
\ddot{r}=\left[h r^{\prime \prime}+r^{\prime} h^{\prime}-\frac{2 h\left(r^{\prime}\right)^{2}}{r}\right] \frac{h}{r^{4}} .
\end{gathered}
$$

Therefore, with (7) and (1), the dynamic equations of the new variables $\left(y_{1}, y_{2}, y_{3}\right)$ are

$$
\begin{gathered}
y_{1}^{\prime}=y_{2}, \\
y_{2}^{\prime}=-y_{1}+y_{3}-\frac{y_{3}}{\mu y_{1}^{2}} u_{r}-\frac{y_{2} y_{3}}{\mu y_{1}^{3}} u_{\theta}, \\
y_{3}^{\prime}=-\frac{2 y_{3}^{2}}{\mu y_{1}^{3}} u_{\theta} .
\end{gathered}
$$

For further simplification, write (8) in matrix form, set $\mathbf{y}=\left(y_{1}, y_{2}, y_{3}\right)^{T}$ and define

$$
\begin{gathered}
A=\left(\begin{array}{ccc}
0 & 1 & 0 \\
-1 & 0 & 1 \\
0 & 0 & 0
\end{array}\right), \quad B=\left(\begin{array}{ll}
0 & 0 \\
1 & 0 \\
0 & 1
\end{array}\right), \\
B_{s}(\mathbf{y})=\left(\begin{array}{rr}
-\frac{y_{3}}{\mu y_{1}^{2}} & -\frac{y_{2} y_{3}}{\mu y_{1}^{3}} \\
0 & -\frac{2 y_{3}^{2}}{\mu y_{1}^{3}}
\end{array}\right), \quad \boldsymbol{v}=B_{s}(\mathbf{y}) \mathbf{u} .
\end{gathered}
$$
is

Thus, state space representation (SSR) of the ODEs in (8)

$$
\mathbf{y}^{\prime}=A \mathbf{y}+B v
$$

The new boundaries of a transfer in the new dynamic equations will be defined by the initial polar angle $\theta_{0}$, the final polar angle $\theta_{1}$ and two constant vectors of the new states

$$
\mathbf{y}\left(\theta_{0}\right)=\mathbf{y}_{0}, \quad \mathbf{y}\left(\theta_{f}\right)=\mathbf{y}_{f}
$$

Under the new state vector $\mathbf{y}$ and new control vector $v$, the objective function, (3), becomes $\left(t^{\prime}(\mathbf{y})=1 / y_{1}^{2} \sqrt{y_{3} / \mu}\right)$

$$
J(\mathbf{u}(\mathbf{y}, \boldsymbol{v}))=J(\mathbf{y}, \boldsymbol{v})=\frac{1}{2} \int_{\theta_{0}}^{\theta} t^{\prime}(\mathbf{y}) \boldsymbol{v}^{T}\left(B_{s}(\mathbf{y}) B_{s}(\mathbf{y})^{T}\right)^{-1} \boldsymbol{v} d \theta
$$

The objective function, (12), dynamic equations, (10) and the boundary conditions, (11), compose the new formulation of the coplanar power-limited orbit transfer problem.

Mathematically, a coplanar power-limited orbit transfer problem can be described as

$$
\text { find: } \quad \begin{aligned}
\boldsymbol{\nu}^{*} & =\arg \min _{\boldsymbol{v}} J(\mathbf{y}(\boldsymbol{v}), \boldsymbol{v}) \\
& =\arg \min _{\boldsymbol{v}} \frac{1}{2} \int_{\theta_{0}}^{\theta} t^{\prime} \boldsymbol{\nu}^{T}\left(B_{s}(\mathbf{y}) B_{s}(\mathbf{y})^{T}\right)^{-1} \boldsymbol{v} d \theta
\end{aligned}
$$

subject to: $\mathbf{y}^{\prime}=A \mathbf{y}+B \boldsymbol{v}$;

$$
\begin{aligned}
& \mathbf{y}\left(\theta_{0}\right)=\mathbf{y}_{0} ; \\
& \mathbf{y}\left(\theta_{f}\right)=\mathbf{y}_{f} .
\end{aligned}
$$

To solve this problem, set $K$ to be the new augmented Hamiltonian and $\boldsymbol{\kappa}$ to be the new adjoint vector corresponding to the new state variable $y$

$$
K=-\frac{1}{2} t^{\prime}(\mathbf{y}) \boldsymbol{\nu}^{T}\left(B_{s}(\mathbf{y}) B_{s}(\mathbf{y})^{T}\right)^{-1} \boldsymbol{v}+\boldsymbol{\kappa}^{T}(A \mathbf{y}+B \boldsymbol{v}) .
$$

Apply the theory of optimal control and the optimal control of the new formulation becomes $\nu^{*}=1$, in which 1 is the primer vector of the new formulation and

$$
\mathbf{l}=\frac{1}{t^{\prime}} B_{s}(\mathbf{y}) B_{s}(\mathbf{y})^{T} B^{T} \boldsymbol{\kappa} .
$$

The mapping from the new primer vector 1 to the original primer vector $\mathbf{p}$ is

$$
\mathbf{p}=B_{s}(\mathbf{y})^{-1} \mathbf{l}
$$

Then the adjoint equations are

$$
\frac{\partial K}{\partial \mathbf{y}}=-\boldsymbol{\kappa}^{\prime}=-\frac{1}{2} \frac{\partial\left(t^{\prime}(\mathbf{y}) \nu^{T}\left(B_{s}(\mathbf{y}) B_{s}(\mathbf{y})^{T}\right)^{-1} \boldsymbol{\nu}\right)}{\partial \mathbf{y}^{T}}+A^{T} \boldsymbol{\kappa} .
$$


In conclusion, the transfer problem given by (13) is an alternative formulation of the coplanar power-limited orbit transfer problem. The optimal solution for this new formulation needs to satisfy a TPBVP defined by the dynamic equation, (10), adjoint equation, (17), the boundary conditions, (11).

2.3. Properties of the New Formulation. The new formulation has unique properties that facilitate analysis. Firstly, the system can be solved explicitly when there is no control $(\boldsymbol{\nu}=0)$ on the state equation, (10). The solutions are

$$
\begin{gathered}
\mathbf{y}(\theta)=\Phi\left(\theta-\theta_{0}\right) \mathbf{y}\left(\theta_{0}\right), \\
\boldsymbol{\kappa}(\theta)=\Phi^{T}\left(\theta_{0}-\theta\right) \boldsymbol{\kappa}\left(\theta_{0}\right), \\
\Phi(\theta)=e^{A \theta}
\end{gathered}
$$

Secondly, the nonlinear term in the adjoint vector equation (17) has the following interesting property.

Theorem 1. For an optimal trajectory, the solution for the adjoint vector has the following form:

$$
\boldsymbol{\kappa}(\theta)=\Phi\left(\theta_{0}-\theta\right)^{T}\left[\mathbf{Q}(\theta)+\mathbf{I}_{3} \times 3\right] \boldsymbol{\kappa}\left(\theta_{0}\right),
$$

where $\theta_{0}$ is the initial polar angle of the trajectory, $\mathbf{I}_{3} \times 3$ is a 3 by 3 unit matrix, and $\mathbf{Q}\left(\theta-\theta_{0}\right)$ is a diagonal matrix function.

Proof. Since (17) is a linear nonhomogeneous equation with constant coefficients, its solution is the sum of the general solution for the related homogeneous equation and the particular integral. The solution can be written down as

$$
\begin{aligned}
\boldsymbol{\kappa}(\theta)= & \Phi\left(\theta_{0}-\theta\right)^{T} \int_{\theta_{0}}^{\theta} \frac{1}{2} \frac{\partial\left(t^{\prime} \boldsymbol{\nu}^{T}\left(B_{s}(\mathbf{y}) B_{s}(\mathbf{y})^{T}\right)^{-1} \boldsymbol{\nu}\right)}{\partial \mathbf{y}^{T}} \Phi(\theta)^{T} d \theta \\
& +\Phi\left(\theta_{0}-\theta\right)^{T} \boldsymbol{\kappa}\left(\theta_{0}\right) .
\end{aligned}
$$

Set

$$
\begin{aligned}
& \left(q_{1}(\theta), q_{2}(\theta), q_{3}(\theta)\right) \\
& \quad=\int_{\theta_{0}}^{\theta} \frac{1}{2} \frac{\partial\left(t^{\prime} \boldsymbol{v}^{T}\left(B_{s}(\mathbf{y}) B_{s}(\mathbf{y})^{T}\right)^{-1} \boldsymbol{v}\right)}{\partial \mathbf{y}^{T}} \Phi(\theta)^{T} d \theta
\end{aligned}
$$

and (use index notation for $\boldsymbol{\kappa}\left(\theta_{0}\right)$ )

$$
\mathbf{Q}(\theta)=\left(\begin{array}{ccc}
\frac{q_{1}(\theta)}{\left(\boldsymbol{\kappa}\left(\theta_{0}\right)\right)_{1}} & 0 & 0 \\
0 & \frac{q_{2}(\theta)}{\left(\boldsymbol{\kappa}\left(\theta_{0}\right)\right)_{2}} & 0 \\
0 & 0 & \frac{q_{3}(\theta)}{\left(\boldsymbol{\kappa}\left(\theta_{0}\right)\right)_{3}}
\end{array}\right) .
$$

With (20), (21), and (22), we obtain (19) of Theorem 1.
Up to this point, no assumption has been made about the new formulation of the transfer problem. It is, however, easy to see that, if the thrust level $|\boldsymbol{\nu}|$ is very small, $\left(q_{1}(\theta), q_{2}(\theta), q_{3}(\theta)\right)$ will become very small. Therefore, for a low-thrust transfer, the adjoint vector can be approximated by setting $\mathbf{Q}(\theta)=\mathbf{0}_{3} \times 3$.

\section{Primer Vector Approximation Method}

3.1. Equivalent Optimal Control Problem. The primer vector approximation method aims to find an approximation for the optimal control (primer vector function), (15), without affecting the accuracy of the state dynamics. Thus, it is important to ensure that the approximate control will lead to a precise integration of the state equation, (10). This is the core of the primer vector approximation method.

To introduce the strategy for primer vector approximation, first define a family of simpler optimal control problems $\Xi$

$$
\text { find: } \quad \hat{\boldsymbol{v}}=\arg \min _{\hat{\boldsymbol{v}}} \hat{J}(\hat{\boldsymbol{v}})=\arg \min _{\hat{\boldsymbol{v}}} \frac{1}{2} \int_{\theta_{0}}^{\theta} \hat{\boldsymbol{\nu}}^{T} C(\theta)^{-1} \hat{\boldsymbol{v}} d \theta
$$

subject to: $\quad \hat{\mathbf{y}}^{\prime}=A \hat{\mathbf{y}}+B \hat{\boldsymbol{v}}$;

$$
\begin{aligned}
& \hat{\mathbf{y}}\left(\theta_{0}\right)=\mathbf{y}_{0} \\
& \hat{\mathbf{y}}\left(\theta_{f}\right)=\mathbf{y}_{f} \\
& C(\theta) \in \chi
\end{aligned}
$$

where $\chi$ is a set of $2 \times 2$ matrix functions and all of its members are continuous while $\theta \in\left[\theta_{0}, \theta_{f}\right]$. The following theorem gives the relationship between the family $\Xi$ and the optimal control problem, defined in (13).

Theorem 2. Given a transfer problem A, defined by (13), among the family of optimal control problem $\Xi$, defined by (23), there is at least one member problem $\hat{A}$ with $C(\theta) \in \chi$ that has the same solution, that is, $\hat{\boldsymbol{v}}^{*}(\theta)=\boldsymbol{v}^{*}(\theta), \hat{\mathbf{y}}^{*}(\theta)=$ $\mathbf{y}^{*}(\theta)$.

Proof. Suppose that the optimal solution for the given problem $A$, defined by (13), is known and set $D(\theta)=$ $1 / t^{\prime}\left(\mathbf{y}^{*}(\theta)\right) B_{s}\left(\mathbf{y}^{*}(\theta)\right) B_{s}\left(\mathbf{y}^{*}(\theta)\right)^{T}$. With Theorem 1, the optimal primer vector function 1 can be written as an explicit function of $\theta$

$$
\mathbf{l}=D(\theta) B^{T} \Phi\left(\theta_{0}-\theta\right)^{T}\left[\mathbf{Q}(\theta)+\mathbf{I}_{3 \times 3}\right] \boldsymbol{\kappa}\left(\theta_{0}\right) .
$$

Set $\boldsymbol{v}=\mathbf{l}$ and plug it into the state equation of (13). We obtain the following relationships for an optimal solution to problem $A$ :

$$
\begin{gathered}
\mathbf{y}^{*}(\theta)=\Phi\left(\theta-\theta_{0}\right)\left(\mathbf{y}_{0}+\Psi(\theta) \boldsymbol{\kappa}_{0}\right), \\
\Psi(\theta)=\int_{\theta_{0}}^{\theta_{1}} \Phi\left(\theta_{0}-\theta\right) B D(\theta) B^{T} \Phi\left(\theta_{0}-\theta\right)^{T}\left[\mathbf{Q}(\theta)+\mathbf{I}_{3 \times 3}\right] d \theta \\
\boldsymbol{\kappa}_{0}=\left(\Phi\left(\theta_{1}-\theta_{0}\right) \Psi\left(\theta_{1}-\theta_{0}\right)\right)^{-1}\left(\mathbf{y}_{f}-\Phi\left(\theta_{1}-\theta_{0}\right) \mathbf{y}_{0}\right) .
\end{gathered}
$$


Meanwhile, any member problem in $\Xi$ can be solved by finding the solution for the following TPBVP:

$$
\begin{aligned}
\hat{\boldsymbol{\kappa}}^{\prime} & =-A^{T} \hat{\boldsymbol{\kappa}}, \\
\hat{\mathbf{y}}^{\prime} & =A \hat{\mathbf{y}}+B \hat{\mathbf{l}}, \\
\hat{\mathbf{y}}\left(\theta_{0}\right) & =\mathbf{y}_{0} ; \hat{\mathbf{y}}\left(\theta_{f}\right)=\mathbf{y}_{f} .
\end{aligned}
$$

This solution is

$$
\begin{aligned}
\hat{\mathbf{y}}^{*}(\theta) & =\Phi\left(\theta-\theta_{0}\right)\left(\mathbf{y}_{0}+\hat{\Psi}(\theta) \hat{\boldsymbol{\kappa}}_{0}\right), \\
\hat{\mathbf{l}}(\theta) & =C(\theta) B^{T} \Phi\left(\theta_{0}-\theta\right)^{T} \hat{\boldsymbol{\kappa}}\left(\theta_{0}\right), \\
\hat{\Psi}(\theta) & =\int_{\theta_{0}}^{\theta_{1}} \Phi\left(\theta_{0}-\theta\right) B C\left(\theta_{0}-\theta\right) B^{T} \Phi\left(\theta_{0}-\theta\right)^{T} d \theta, \\
\hat{\boldsymbol{\kappa}}_{0} & =\left(\Phi\left(\theta_{1}-\theta_{0}\right) \hat{\Psi}\left(\theta_{1}\right)\right)^{-1}\left(\mathbf{y}_{f}-\Phi\left(\theta_{1}-\theta_{0}\right) \mathbf{y}_{0}\right) .
\end{aligned}
$$

Select a problem $\hat{\mathscr{A}} \in \Xi$ with

$$
C(\theta)=D(\theta) B^{T} \Phi\left(\theta_{0}-\theta\right)^{T}\left[\mathbf{Q}(\theta)+\mathbf{I}_{3 \times 3}\right] \Phi\left(\theta-\theta_{0}\right)^{T} B .
$$

Put (28) into (27). After simplification, we obtain

$$
\begin{aligned}
\hat{\Psi}(\theta) & =\Psi(\theta), \\
\hat{\boldsymbol{\kappa}}_{0} & =\boldsymbol{\kappa}_{0} .
\end{aligned}
$$

Therefore, the optimal control and optimal trajectory of problems $A$ and $\hat{A}$ are the same, that is,

$$
\hat{\boldsymbol{v}}^{*}(\theta)=\boldsymbol{v}^{*}(\theta), \quad \hat{\mathbf{y}}^{*}(\theta)=\mathbf{y}^{*}(\theta) .
$$

If $\hat{\mathcal{A}} \in \Xi$ and a transfer problem $\mathcal{A}$ has the same solution, we call $\hat{\mathscr{A}}$ the equivalent optimal control problem of the original problem $\mathcal{A}$ in (13).

Theorem 3. If $\hat{A} \in \Xi$ is an equivalent optimal control problem of a transfer problem $\mathcal{A}$, defined by (13), then the optimal control $\hat{\mathbf{1}}$ of another member problem $\widehat{\mathscr{B}} \in \Xi$ is a feasible control for the transfer problem $\mathcal{A}$.

Proof. From the general solution equation (27), we know that no matter what functional form of $C(\theta)$ we pick, the obtained control $\hat{\mathbf{l}}$ will always generate a trajectory that satisfies the two end point boundaries. Thus, $\hat{\mathbf{l}}$ is a feasible control for the power-limited transfer problem.

Theorem 2 gives us a new way to solve any optimal power-limited transfer problem $\mathcal{A}$. That is, we first find its equivalent problem in $\widehat{\mathcal{A}} \in \Xi$, and then the optimal solution is given by $\mathbf{y}(\theta)=\hat{\mathbf{y}}(\theta), \mathbf{l}(\theta)=\hat{\mathbf{l}}(\theta)$, and (27). In fact, from the proof of Theorem 2, we can construct such a problem $\hat{\mathcal{A}} \in \Xi$ with $C(\theta)=C^{*}(\theta)$,

$$
\begin{gathered}
C^{*}(\theta)=D(\theta) B^{T} \Phi\left(\theta_{0}-\theta\right)^{T}\left[\mathbf{Q}(\theta)+\mathbf{I}_{3 \times 3}\right] \Phi\left(\theta-\theta_{0}\right)^{T} B, \\
C^{*}\left(\theta_{0}\right)=D\left(\theta_{0}\right) .
\end{gathered}
$$

Though we do not really know $D(\theta)$ until we solve the original problem $\mathcal{A}$, we can always "shape" the matrix function $C(\theta)$ ) based on our assumptions and knowledge of the transfer scenario. Suppose the "shaped" matrix function is $C^{\circ}(\theta)$ and it corresponds to a problem $\widehat{\mathscr{B}} \in \Xi$. Theorem 3 indicates that, even though $\widehat{\mathscr{B}}$ is not exactly $\widehat{A}$, it gives a feasible solution for the original problem $\mathcal{A}$ as long as $\widehat{\mathscr{B}} \in \Xi$. Thus, if we can find a proper $C^{\circ}(\theta)$ satisfying $C^{\circ}(\theta) \approx C^{*}(\theta)$, then the solution for $\widehat{\mathscr{B}},(27)$, is a feasible approximate solution for $\hat{A}$, essentially $\mathcal{A}$. In other words, once we find $C^{\circ}(\theta)$ (an approximation of $C^{*}(\theta)$ ), the approximate solution to $\mathcal{A}$ is given by (27) with $C(\theta)=C^{\circ}(\theta)$. Since any $C(\theta) \in \chi$ corresponds to a unique primer vector function $\hat{\mathbf{l}}(\theta)$ in (27), this process of finding an approximation of $C^{*}(\theta)$ is named as primer vector approximation (PVA) in this paper. The following section uses this PVA to derive two explicit approximate solutions to the coplanar power-limited orbit transfer problem.

3.2. Primer Vector Approximation under the Low-Thrust Assumption. In this section, we assume the thrusting magnitude is very low (i.e., $|\boldsymbol{v}|$ is very small), such that $\mathbf{Q}(\theta) \approx$ $\mathbf{0}_{3 \times 3}, C^{*}(\theta)$ becomes a symmetric matrix function and

$$
C^{*}(\theta) \approx \frac{1}{t^{\prime}\left(\mathbf{y}^{*}(\theta)\right)} B_{s}\left(\mathbf{y}^{*}(\theta)\right) B_{s}\left(\mathbf{y}^{*}(\theta)\right)^{T}
$$

The thrusting magnitude is usually very low in two kinds of transfers. One is the close range transfer and the other the long duration multirevolution transfer.

3.2.1. Approximate Solution for Close Range Transfer. Because the transfer happens in the vicinity of the initial orbit, a reasonable approximation of $C^{*}(\theta)$ is

$$
\begin{gathered}
C^{\circ}(\theta)=\frac{1}{t^{\prime}\left(\mathbf{y}^{0}(\theta)\right)} B_{s}\left(\mathbf{y}^{0}(\theta)\right) B_{s}\left(\mathbf{y}^{0}(\theta)\right)^{T}, \\
\mathbf{y}^{0}(\theta)=\Phi\left(\theta-\theta_{0}\right) \mathbf{y}_{0},
\end{gathered}
$$

where $\mathbf{y}^{0}(\theta)$ represents the initial orbit. Put (33) into (27). We obtain the approximate optimal solution for a close range transfer.

Though (27) generally requires numerical integration to get $\Psi$, the approximate optimal trajectory $y^{*}$ and control $\boldsymbol{\nu}^{*}$ are still explicit functions of $\theta$. Moreover, $\Psi$ is independent of the target location $\mathbf{y}_{1}$. Therefore, for all possible closerange transfers of the initial orbit $\mathbf{y}_{0}(\theta)$, we only need to compute $\Psi$ one time for one period of the initial orbit. This property is greatly useful when we schedule transfers for a satellite formation around an elliptic reference orbit specified by $\mathbf{y}_{0}$. 
TABLE 1: Numerical tests of approximate solutions.

\begin{tabular}{lcccccccc}
\hline \multirow{2}{*}{ Case } & Initial Orb. & Target Orb. & \multicolumn{2}{c}{ Approx. C.R. } & \multicolumn{2}{c}{ Approx. L.D. } & \multicolumn{2}{c}{ Optimum } \\
& $\left(a_{0}, e_{0}, \theta_{0}\right)$ & $\left(a_{1}, e_{1}, \theta_{1}\right)$ & TOF & Cost $J(\mathbf{u})$ & TOF & Cost $J(\mathbf{u})$ & TOF & Cost $J(\mathbf{u})$ \\
\hline A $(<1$ Rev $)$ & $(1,0,0)$ & $(1,0.1,4)$ & 4.364 & 0.0009474 & 4.360 & 0.0009469 & 4.359 & 0.0009424 \\
B (<1 Rev) & $(1,0.8,0)$ & $(1,0.85,4)$ & 6.026 & 0.0005230 & 5.962 & 0.007268 & 6.016 & 0.0005148 \\
C (=4 Revs $)$ & $(1,0,0)$ & $(2,0.15,8 \pi)$ & 41.48 & 0.002301 & 42.26 & 0.002314 & 41.28 & 0.002290 \\
D (=20 Revs $)$ & $(1,0,0)$ & $(2,0,40 \pi)$ & 208.2 & 0.0004287 & 202.3 & 0.0004288 & 205.1 & 0.0004277 \\
\hline
\end{tabular}

When the initial orbit is circular, the computation of $\Psi$ can be done analytically. Using index notation and setting $\Delta \theta=\theta_{f}-\theta_{0}, c=\cos (\Delta \theta)$ and $s=\sin (\Delta \theta)$, we have

$$
\begin{aligned}
& (\Psi)_{11}=\frac{1}{2 \sqrt{\left(\mathbf{y}_{0}\right)_{1}}}(-16+3 c) s+13 \Delta \theta \\
& (\Psi)_{12}=(\Psi)_{21}=\frac{1}{4 \sqrt{\left(\mathbf{y}_{0}\right)_{1}}}\left(-13+16 c-3 c^{2}+3 s^{2}\right) \\
& (\Psi)_{13}=(\Psi)_{31}=\frac{4}{\sqrt{\left(\mathbf{y}_{0}\right)_{1}}}(-s+\Delta \theta) \\
& (\Psi)_{22}=\frac{1}{2 \sqrt{\left(\mathbf{y}_{0}\right)_{1}}}(-3 c \times s+5 \Delta \theta) \\
& (\Psi)_{23}=(\Psi)_{32}=(\Psi)_{33}=\frac{4}{\sqrt{\left(\mathbf{y}_{0}\right)_{1}}}(-1+c) .
\end{aligned}
$$

With (34), the approximate optimal solution, (27), becomes analytic.

3.2.2. Approximate Solution for Long Duration Multirevolution Transfer. A long duration multirevolution transfer tends to build up its orbital energy and angular momentum monotonically. Thus, it is reasonable to approximate the characteristic matrix function $C^{*}(\theta)$ with a linear matrix function

$$
\begin{aligned}
C^{\circ}(\theta) & =C^{\circ}\left(\theta_{0}\right)+\frac{\theta-\theta_{0}}{\theta_{f}-\theta_{0}}\left[C^{\circ}\left(\theta_{f}\right)-C^{\circ}\left(\theta_{0}\right)\right], \\
C^{\circ}\left(\theta_{0}\right) & =\frac{1}{t^{\prime}\left(\mathbf{y}_{0}\right)} B_{s}\left(\mathbf{y}_{0}\right) B_{s}\left(\mathbf{y}_{0}\right)^{T}, \\
C^{\circ}\left(\theta_{f}\right) & =\frac{1}{t^{\prime}\left(\mathbf{y}_{f}\right)} B_{s}\left(\mathbf{y}_{f}\right) B_{s}\left(\mathbf{y}_{f}\right)^{T} .
\end{aligned}
$$

With (35), the matrix function $\Psi$ in (27) can be expanded analytically. With the analytic $\Psi$, the approximate optimal solution given by (27) is analytic. (The complete formula for $\Psi$ is too lengthy to show here.)

\section{Numerical Test}

4.1. Cases of Free-Time Rendezvous Problem. Without loss of generality, we can set the gravitation constant $\mu=1$ and use normalized distance in the test cases.

In Table 1, four coplanar rendezvous cases are chosen to test the accuracy of the two analytic approximations. Cases A and $\mathrm{B}$ test the approximate solutions for close range transfers, with case A featuring a circular reference while case B featuring a high elliptic reference. Cases C and D test the approximate solutions for long duration transfers. Case $\mathrm{C}$ is a circular-to-elliptic transfer, while case D is a circularto-circular transfer. We use "Approx. C.R." to identify the approximate solution for close range transfer, (27) and (23), and use "Approx. L.D." to identify the approximate solution for long range transfer, (27) and (35).

4.2. Results and Discussions. The results of cases A and B show that "Approx. C.R" captures the close-range-transfer primer vector dynamics. Where the circular reference is used (case A), the percentage error of the cost and the time of flight (TOF) are about $0.5 \%$ and $0.1 \%$, respectively. As the eccentricity of the reference orbit increases, the primer vector dynamics becomes more complicated. However, even when the eccentricity of the reference orbit is as high as 0.8 (case B), "Approx. C.R" still captures the primer vector dynamics well. The percentage error of the cost and the TOF are about $1.5 \%$ and $0.16 \%$, respectively.

The results of cases C and D show that "Approx. L.D" captures the long-duration-transfer primer vector dynamics. The percentage error of the cost and the time of flight (TOF) are around $1 \%$ and $2.3 \%$, respectively, for case $\mathrm{C}$, and around $0.26 \%$ and $1.4 \%$, respectively, for case D.

It is interesting to see that "Approx. C.R", intended for close-range transfers, works very well for long-duration transfers too. It is, however, worth pointing out that, since "Approx. C.R" requires a numerical integration, when the initial orbit is elliptic, it is computationally slower than "Approx. L.D." But when the initial orbit is circular, the analytic "Approx. C.R" actually becomes computationally faster than "Approx. L.D."

A significant advantage of the primer vector approximation method is that it precisely follows the dynamic equations of motion and gives a feasible solution. Table 2 shows the missed target errors of case $\mathrm{D}$, which are obtained by simulating Equations (2) with the generated control profiles of the two approximations. The results verify the advantage of the proposed method.

Figures 2 and 3 show the thrust angle and thrust magnitude histories of case B, while Figures 5 and 6 show those of case D. Figures 4 and 7 present the trajectories for case B and case $\mathrm{D}$, respectively. In the figures, the black solid line represents the optimal solution, while the black dashed line is from "Approx. LD" and the gray solid line from "Approx. CR". Only the "Approx. CR" solution (gray line) and the true 
TABLE 2: Errors induced by the approximate solutions (case D).

\begin{tabular}{lcccc}
\hline & $r$ & $\theta$ & $v_{r}$ & $v_{\theta}$ \\
\hline Approx. CR & $-1.51 \times 10^{-7}$ & $-9.77 \times 10^{-6}$ & $-1.02 \times 10^{-6}$ & $7.66 \times 10^{-7}$ \\
Approx. LD & $-4.47 \times 10^{-7}$ & $-7.13 \times 10^{-6}$ & $-8.00 \times 10^{-7}$ & $1.73 \times 10^{-7}$ \\
\hline
\end{tabular}

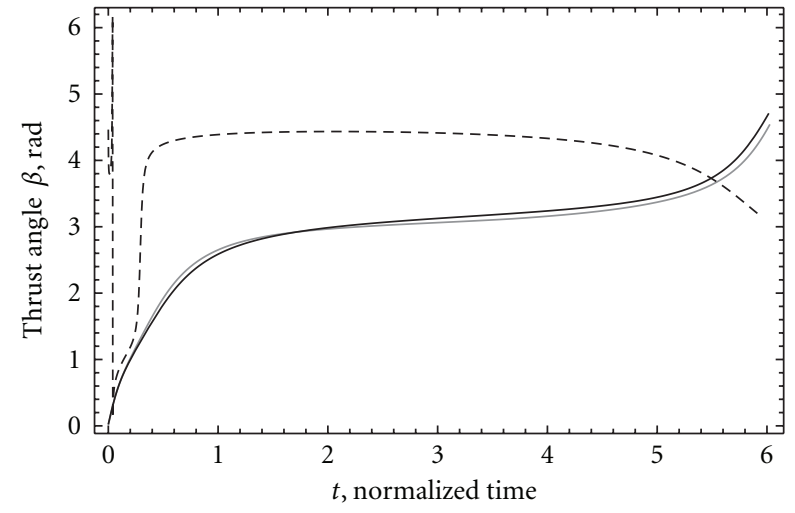

FIgURE 2: Thrust angle comparison of different trajectories in case B.

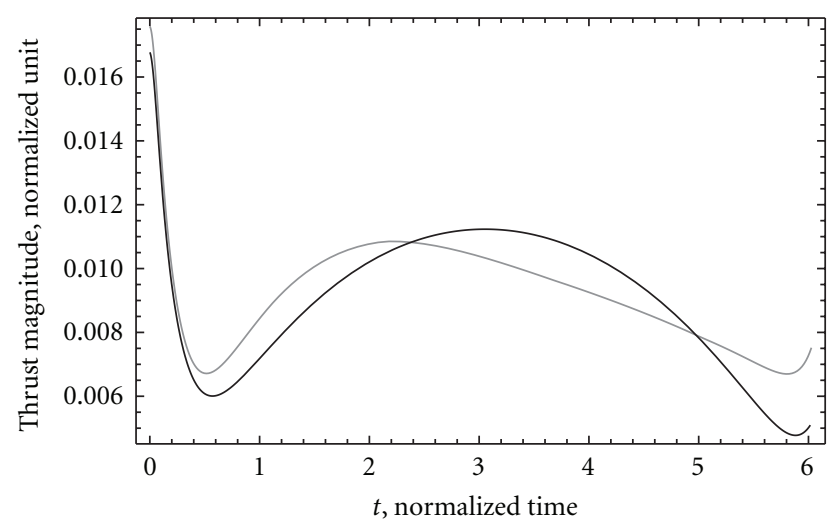

Figure 3: Thrust magnitude comparison of different trajectories in case B.

optimal solution are shown in Figure 3, because the "Approx. LD" solution is too far away from the optimum to effectively display.

\section{Conclusions}

This paper uses a new method to approximate solutions for a nonlinear optimal control problem. This method begins with a transformation to push all the nonlinearity to the coefficients of the control terms. Then it analyzes of the adjoint equations and embeds the process of finding solutions into the process of approximating the primer vector curve of the equivalent linear quadratic optimal control problem. This method is powerful and leads to an extremely simple and accurate explicit approximate solution. At the cases of circular close-range transfers and long-duration transfers, analytic approximate solutions exist.

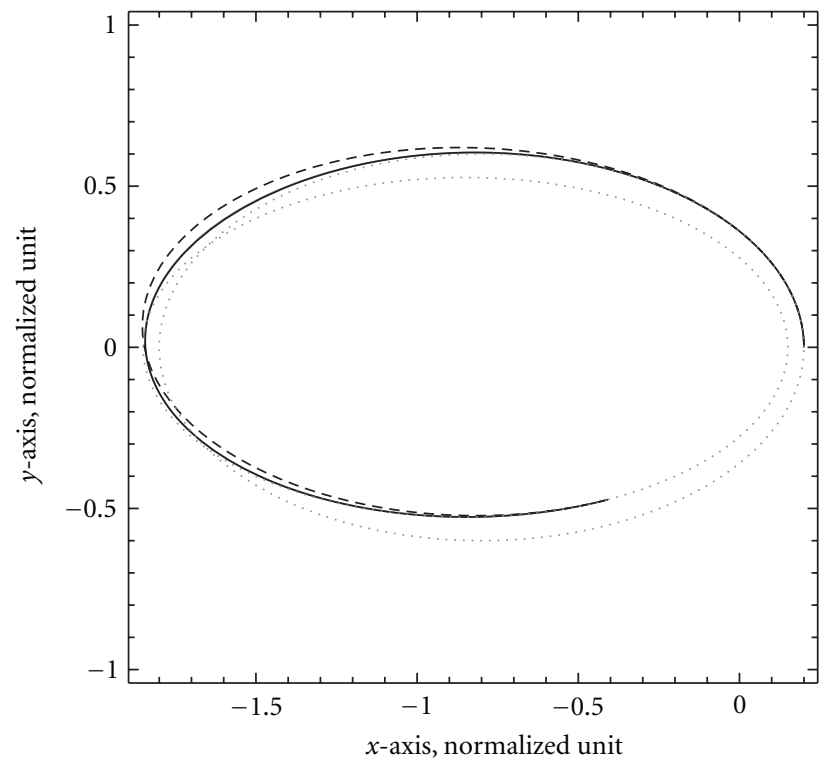

FIgURE 4: Visual comparison of different trajectories in case B.

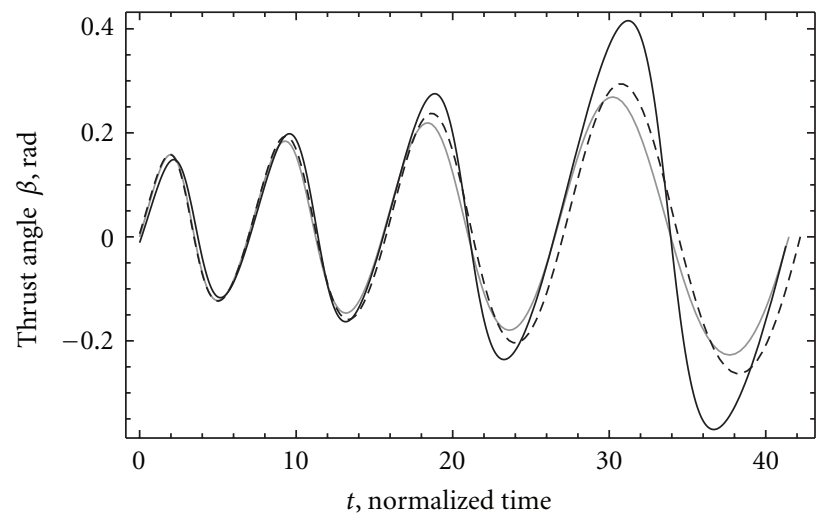

FIGURE 5: Thrust angle comparison of different trajectories in case D.

This paper focuses on the display of the primer vector approximation method itself and includes no numerical comparison of this new method with other approximation methods. However, as far as the author knows, the proposed method is quite unique and radically different from other approximation methods. This method has a precise integration of the state dynamics, while other approximation methods more or less approximate the state dynamics. As a result, even though the given transfer scenario largely violates the assumptions of the approximation, the obtained 


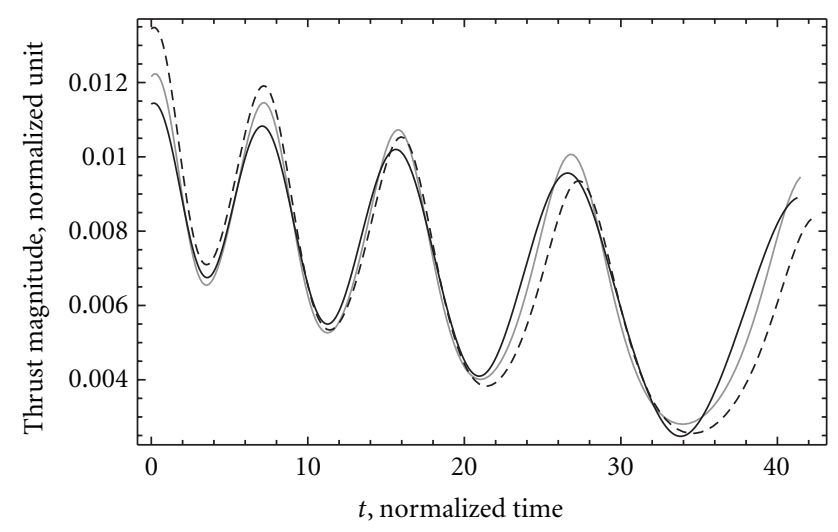

FIGURE 6: Thrust magnitude comparison of different trajectories in case D.

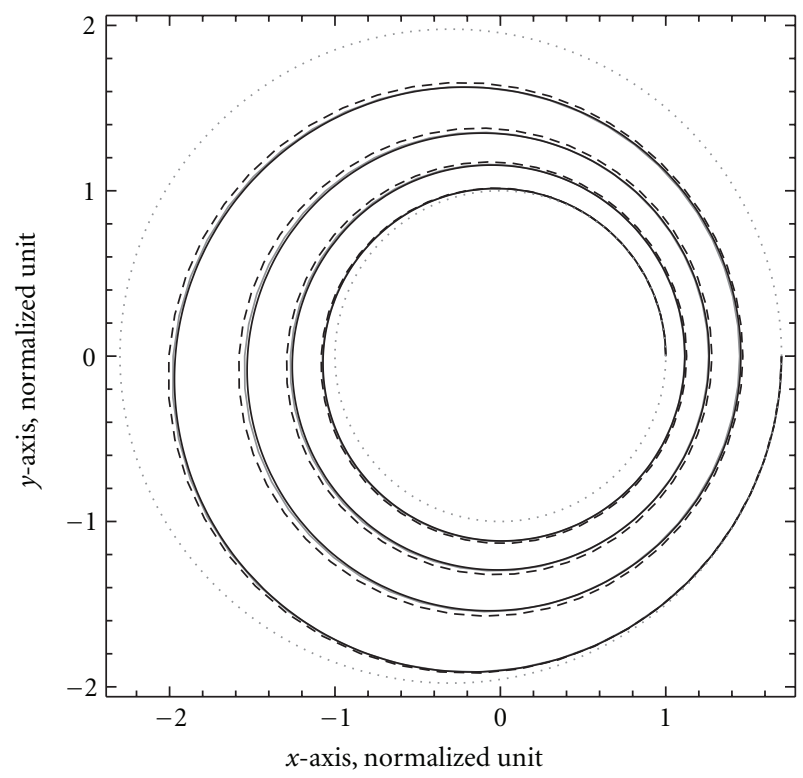

FIGURE 7: Visual comparison of different trajectories in case D.

solutions are still feasible. This is a major advantage of using the primer vector approximation method.

\section{Nomenclature}

$\mu$ : Standard gravitational parameter of the Earth

$r$ : Distance from the attraction center to the spacecraft

$\theta$ : Polar angle of the spacecraft in the polar coordinate

$v_{r}$ : Radial direction component of the velocity of the spacecraft

$v_{\theta}$ : Normal direction component of the velocity of the spacecraft

$\beta$ : Thrust angle of the spacecraft

$\lambda_{r}$ : Adjoint variable corresponding to $r$

$\lambda_{\theta}$ : Adjoint variable corresponding to $\theta$ $\lambda_{v r}:$ Adjoint variable corresponding to $v_{r}$

$\lambda_{v \theta}$ : Adjoint variable corresponding to $v_{\theta}$

$u_{r}$ : Control acceleration in the radial direction of the spacecraft

$u_{\theta}$ : Control acceleration in the normal direction of $u_{r}$

$H$ : Augmented Hamiltonian of the polar coordinate formulation of trajectory optimization

$K$ : Augmented Hamiltonian of the new formulation of trajectory optimization

$J(\mathbf{u})$ : Objective function of the power-limited transfer problem

$\hat{J}(\hat{\boldsymbol{v}})$ : Objective function of the equivalent problem

$y$ : State vector of a space vehicle in the new formulation

$\boldsymbol{\kappa}$ : Adjoint vector of the the new formulation

$\boldsymbol{v}$ : Control variable in the new formulation

l: Primer vector in the new formulation

$\hat{\mathbf{y}}$ : State vector of the equivalent problem

$\hat{\boldsymbol{\kappa}}$ : Adjoint vector of the equivalent problem

$\hat{v}$ : Control variable of the equivalent problem

$\hat{\mathrm{l}}$ : Primer vector of the equivalent problem.

\section{Subscripts}

0: Initial value

$f$ : Final value.

\section{Superscripts}

T: $\quad$ Transpose of a matrix

*: Optimal value

$\square^{\prime}$ : First derivative with respect to the polar angle

$\square$ ": Second derivative with respect to the polar angle.

\section{References}

[1] T. N. Edelbaum, "Theory of maxima and minima," in Optimization techniques: with applications to aerospace systems, G. Leitmann, Ed., vol. 5, chapter 1, Academic Press, 1962.

[2] T. N. Edelbaum, "Optimum low-thrust rendezvous and station keeping," Journal of Spacecraft and Rockets, vol. 40, no. 6, pp. 960-965, 2003.

[3] T. N. Edelbaum, "Optimum power-limited orbit transfer in strong gravity fields," AIAA Journal, vol. 3, pp. 921-925, 1965.

[4] T. N. Edelbaum, "An asymptotic solution for optimum power limited orbit transfer," AIAA Journal, vol. 4, pp. 1491-1494, 1966.

[5] C. Zee, "Low constant tangential thrust spiral trajectories," AIAA Journal, vol. 1, no. 7, pp. 1581-1583, 1963.

[6] A. Marinescu, “Optimal low-thrust orbital rendezvous," Journal of Spacecraft and Rockets, vol. 13, no. 7, pp. 385-392, 1976.

[7] J. P. Marec and N. X. Vinh, "Optimal low-thrust, limited power transfers between arbitrary elliptical orbits," Acta Astronautica, vol. 4, no. 5-6, pp. 511-540, 1977.

[8] C. M. Haissig, K. D. Mease, and N. X. Vinh, "Minimum-fuel, power-limited transfers between coplanar elliptical orbits," Acta Astronautica, vol. 29, no. 1, pp. 1-15, 1993. 
[9] J. A. Kechichian, "Reformulation of Edelbaum's low-thrust transfer problem using optimal control theory," Journal of Guidance, Control, and Dynamics, vol. 20, no. 5, pp. 988-994, 1997.

[10] L. Casalino and G. Colasurdo, "Improved Edelbaum's approach to optimize low earth/geostationary orbits low-thrust transfers," Journal of Guidance, Control, and Dynamics, vol. 30, no. 5, pp. 1504-1510, 2007.

[11] Y. Gao and C. A. Kluever, "Analytic orbital averaging technique for computing tangential-thrust trajectories," Journal of Guidance, Control, and Dynamics, vol. 28, no. 6, pp. 1320-1323, 2005.

[12] N. Markopoulos, "Explicit, near-optimal guidance for powerlimited transfers between coplanar circular orbits," Journal of Guidance, Control, and Dynamics, vol. 19, no. 6, pp. 13171325, 1996.

[13] A. E. Petropoulos and J. M. Longuski, "Shape-based algorithm for automated design of low-thrust, gravity-assist trajectories," Journal of Spacecraft and Rockets, vol. 41, no. 5, pp. 787-796, 2004.

[14] B. J. Wall and B. A. Conway, "Shape-based approach to lowthrust rendezvous trajectory design," Journal of Guidance, Control, and Dynamics, vol. 32, no. 1, pp. 95-102, 2009.

[15] D. M. Novak and M. Vasile, "Improved shaping approach to the preliminary design of low-thrust trajectories," Journal of Guidance, Control, and Dynamics, vol. 34, no. 1, pp. 128-147, 2011.

[16] T. Carter and M. Humi, "Three-impulse rendezvous near circular orbit determined through a new primer vector analysis," in Proceedings of the AAS/AIAA Spaceflight Mechanics Meeting, vol. 140 of Advances in the Astronautical Sciences Series, 2011. 

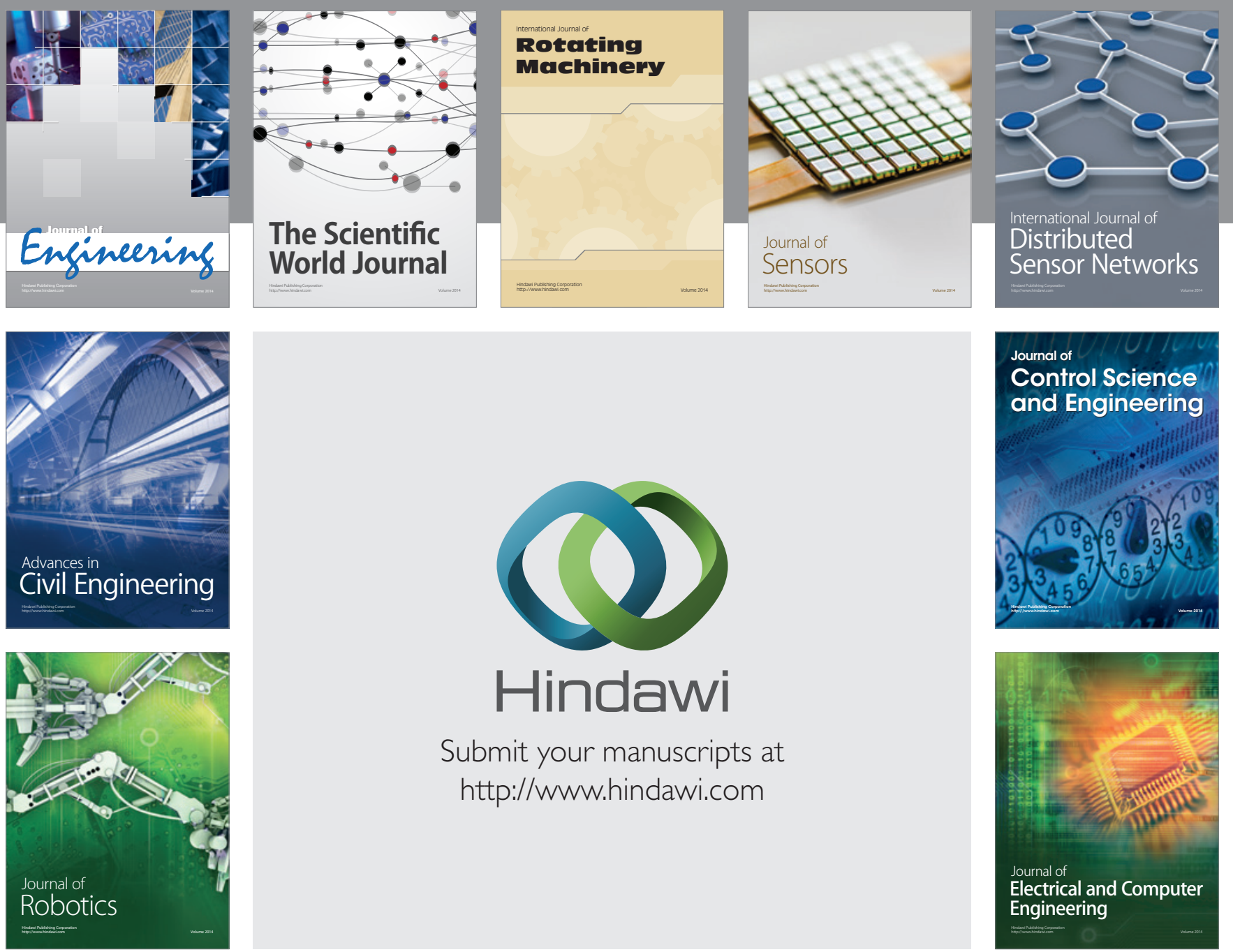

Submit your manuscripts at

http://www.hindawi.com
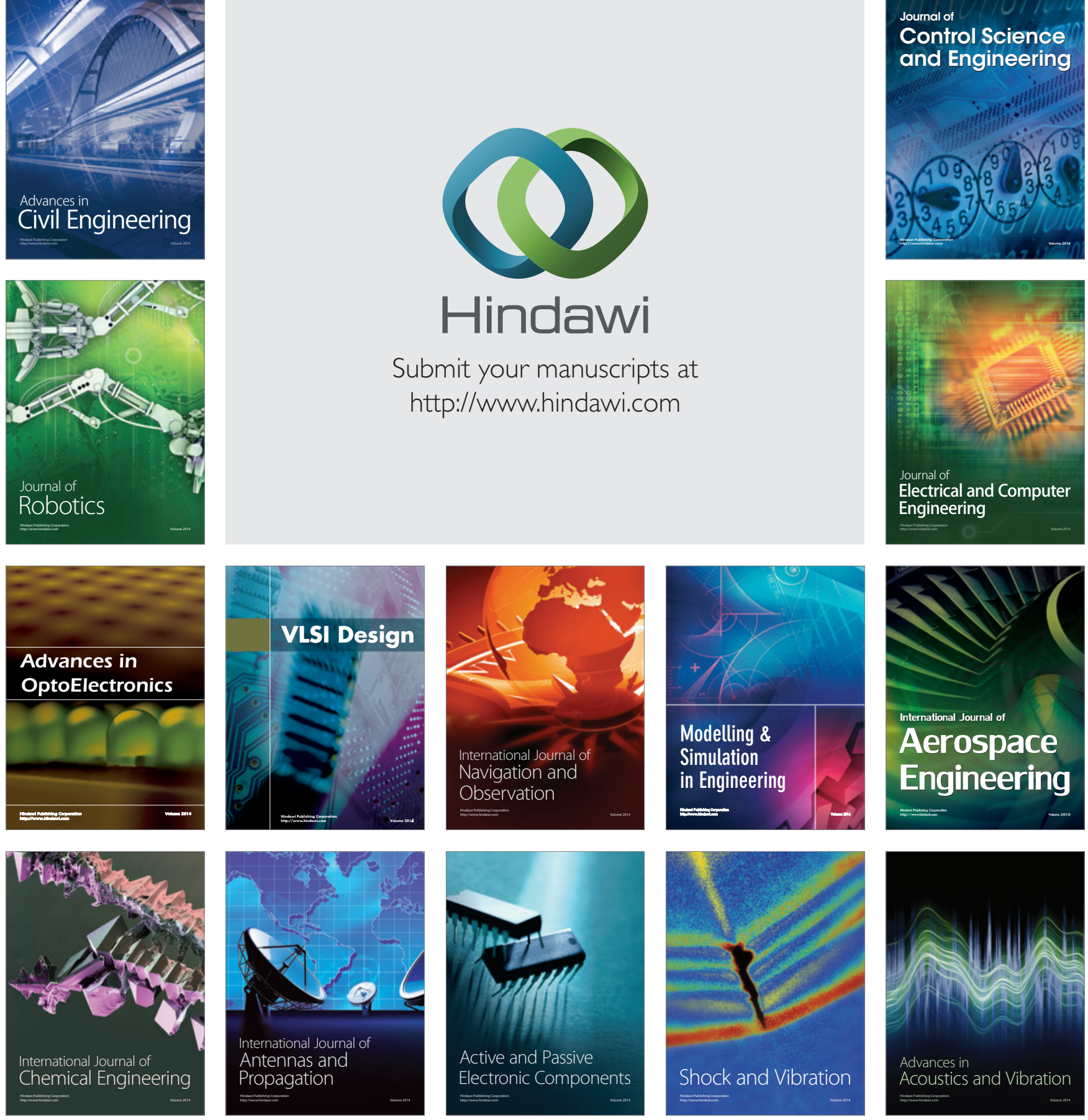